\title{
THE PIONEER
}

for $\mathrm{SB}$

The angry man has learned peace

from the old teacher,

an acre of garden.

Planting the Indian way:

a handful of manure, a handful of clay, soft blankets for each sleeping seed.

In the ripened heat of dusk he sits on the ground, silently laying track within himself,

filling in the blank spaces of his map, and sometimes, ear to the rail, he can feel the sound of something far-off,

coming. 